\title{
Projectile Penetrating Multilayer Composite Armor
}

\author{
P. A. Prasob ${ }^{1}$, M. Sasikumar ${ }^{1 *}$, Navneet Bardiya' ${ }^{1}$ and P. Vasundhra ${ }^{2}$ \\ 'School of Mechanical and Building Sciences (SMBS), VIT University, Chennai Campus, Chennai - 600127, Tamil \\ Nadu, India; pprasob@gmail.com, Sasikumar.m@vit.ac.in, navneet.bardiya2013@vit.ac.in \\ ${ }^{2}$ CVRDE, Defense Research and Development Organization, Avadi, Chennai - 600 054, Tamil Nadu, India; \\ vasundhra.p@cvrde.drdo.in
}

\begin{abstract}
Objectives: The objective of this paper is to study the influence of interface between multi layered composite structures in penetration problems, using finite element solver RADIOSS. Methods/Statistical Analysis: Finite element modeling of the penetrating projectile, multilayer composite and analysis using RADIOSS is done. The analysis is confined to Johnson Cook material model for plasticity and failure. One of the important aspects of material model is the representation of the plasticity and failure region. The modified interface creates a realistic environment with combination of TYPE 2 and TYPE 7 influences multiple hit of projectiles and does not influence the depth of penetration when compared to the TYPE 7 interface. Findings: Hyper Works software tool is used to simulate the projectile penetrating armor plate and Hyper Mesh used for preprocessing and RADIOSS as a solver. The depth of penetration is not significantly affected due to the TYPE 2 interface. However, the delamination behavior of the layers is captured in the TYPE 2 interface when compared to TYPE7. The modified interface with combination of TYPE 2 and TYPE 7 does not influence the depth of penetration when compared to the TYPE 7 interface. TYPE 7 formulations gives considerably acceptable results for capturing the depth of penetration in case of multilayer composite structures. TYPE 2 interfaces has to be studied in cases where delamination has influence, for example in multiple hits of projectiles etc. Application/Improvements: Development of alternative material or sandwich structure is required for the future war scenario to prevent the main threats for Armored Fighting Vehicles (AFVs), shock and penetration as future war scenario insists on light tanks that can withstand penetration with higher order of destruction.
\end{abstract}

Keywords: Armoured Fighting Vehicle (AFV), Finite Element Modeling, 7.62 mm Projectile, Multi Material

\section{Introduction}

Kinetic energy projectile is one of the major threats in the modern war scenario. The effects of this go beyond physical damage to the crew. Hence there is always a need for optimal design of vehicles and structures to withstand penetration and ensure the survivability of the crew. In literaturef, the number of papers discusses on the finite element modeling and analysis of the effect of projectile penetrating sandwich structures. Generally, in case of multi material armour plates, the layers are glued together using resins. In most of the papers, this contact is modeled using general penalty contact or the nodes of the layers are merged. In the first scenario, the analysis is expected to under-estimates the effect of contact which happens in the actual scenario. In the second case, it is equivalent to represent a perfectly welded plate, which is not the actual phenomenon. In this paper, an alternative method of penalty contact is used.

This section gives a brief introduction on material and finite element modeling. One of the important aspects of material model is the representation of the plasticity and failure region. The accuracy of the results depends on the accuracy of the material model. Also, it is to be noted that the mesh elements and size play a significant role in the accuracy of the result, since the explicit analysis are conditionally convergent 


\subsection{Material Models}

Structural materials: In the elastic zone, the stress is proportional to strain and follows the Hooke's law. Beyond the elastic region, the material behaves nonlinearly. Dynamic loads like impact problems typically produce very high strain rates in the range of. This high strain rate dictates the dynamic mechanical properties of the target and, accordingly, the expected damage mechanisms for various structural elements strain is the sum of the elastic and plastic strain. Hence, it is important to model the non-linear behavior of materials. In fact, this greatly influences the outcome of the result. The much finite element software has the option of including the material model in the form of lookup table or simplified empirical formula. The type of approximation depends on the problems and the level of accuracy required. The simplest form of approximation isf

$$
\sigma=K \epsilon^{n}
$$

In the present paper, the analysis is confined to Johnson Cook material model for plasticity and failure. The subsequent sections discuss in brief of the material model.

\subsubsection{Strength Model}

Several constitutive material models, which represent large strain behavior over a wide range of strain rates and temperatures, have been proposed in the literaturef. Examples of such models are Johnson-Cook, ZerilliArmstrong, etc. The Johnson-Cook material model is particularly suited to model high strain rate deformation of metals. It is generally used in the adiabatic transient dynamic analysis. The hardening is a particular type of isotropic hardening in which the stress $\sigma_{0}$ is assumed to be of the form.

$\sigma_{0}=\left(\mathbf{A}+\mathbf{B}\left(\boldsymbol{\varepsilon}^{\mathrm{p}}\right)^{\mathrm{n}}\right)\left(1+\operatorname{Cln}\left(\dot{\varepsilon}_{\mathrm{p}} / \dot{\varepsilon}_{0}\right)(1-) \widehat{\mathrm{T}}_{\mathrm{m}}\right)$

Where

$\widehat{T}_{m}=\left\{\begin{aligned} 0, & T \leq T_{r} \\ \frac{T-T_{r}}{T_{m}-T_{r}}, & T_{r} \leq T \leq T_{m} \\ 1, & T \geq T_{m}\end{aligned}\right.$
In the above expression $\epsilon^{p}$ is the equivalent plastic strain, $\epsilon_{0}$ is the strain rate at reference temperature, $\epsilon^{p}$ is the strain rate, $T_{m}$ is melting temperature and $T_{r}$ is the reference temperature. The parameters $A, B, C, n$ and $m$ are obtained from the material data. Here, $A$ is the yield stress $\sigma_{y}$ at temperatures below $T_{r}, B$ is called the hardening parameter, $n$ the hardening exponent, $C$ is the strain rate constant and $m$ is the temperature exponent? These parameters are obtained from the experimental data.

\subsubsection{Failure Model}

The failure of a material is usually classified into brittle failure (fracture) or ductile failure (yield). Depending on the conditions (such as temperature, state of stress, loading rate) most materials can fail in a brittle or ductile manner or both. However, for most practical situations, a material may be classified as either brittle or ductile. Johnson Cook failure modelis a widely used model which has a cumulative damage law that can access failure:

$d=\sum\left[\frac{\Delta \varepsilon}{\varepsilon_{f}}\right]$

$\varepsilon_{f}=\left[D_{1}+D_{2} \exp \left(D_{3} \sigma^{*}\right)\right]\left[1+D_{4} \ln \left(\frac{\dot{\varepsilon}}{\dot{\varepsilon}_{0}}\right)\right.$.

where $\Delta \varepsilon$ an increment of the equivalent plastic strain is, $\varepsilon_{f}$ is the strain at failure, and the summation is performed over all increments in the analysis. $\sigma^{*}=\frac{\sigma^{m}}{\sigma_{V M}}$ the normalized mean stress and the parameters $D_{i}$ the material constants if. Failure is assumed to occur when $d=1$.

\subsubsection{Finite Element Modeling and Analysis using RADIOSS}

Both projectile and plate are modeled as a deformable. For projectile, 4-node solid linear tetrahedron and for armour 8-node solid linear hexahedrons are considered. For tetrahedron mesh of projectile, hourglass effect does not exist. In the case of 8-node solid hexa mesh, the hour glass modes exist. In order to correct this phenomenon, it is necessary to introduce the anti hourglass effect. RADIOSS is based on explicit solver methods that are widely used for impact problems' 


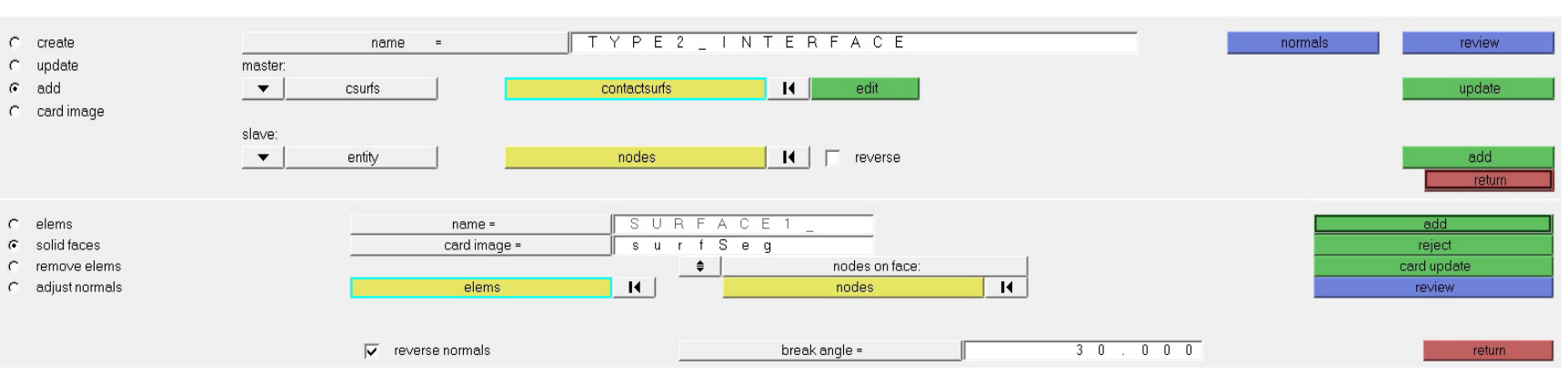

Figure 1. Example of TYPE 2 interface setup.

\subsubsection{Contact treatment}

There are two different methods to define the interaction between two bodies in RADIOSS explicit viz., TYPE 7 interface and TYPE 2 interface.

\subsubsection{TYPE 7 interface}

In RADIOSS, TYPE 7 interface defines a contact between a master surface and a group of slave nodes. It requires interface stiffness definition as well as the deactivation of stiffness in case of initial penetration, gap/element option, node and segment deletion and friction penalty formulation to be defined

\subsubsection{TYPE 2 interfaces}

In RADIOSS, the structural adhesive contact is based on TYPE 2 interface ${ }^{\text {I }}$. The standard TYPE 2 tied interface that kinematically connects a set of slave nodes to a master surface can be used to connect coarse and fine meshes, model spot-weld, rivets, resins etc.

The TYPE 2 interface is governed by additional stress failure criteria in addition to the kinematic condition. The slave layer node is redefined to include the sum of surfaces connected to the already defined slave node. Because of the infinite stiffness of kinematic interface at failure, it becomes necessary to include interface deformation. The option of using Type 2 interface is as shown in Figure 1.

The deformation of elements at the interface layer follows the modified failure criterion based on displacements. The advantage of the displacement failure criterion is based on the small thickness of the physical adhesive layer. Taking into account, the adhesive failure strain, the relation of the displacement of a thin adhesive layer until failure in tension and shear is approximated th $^{\text {. }}$.

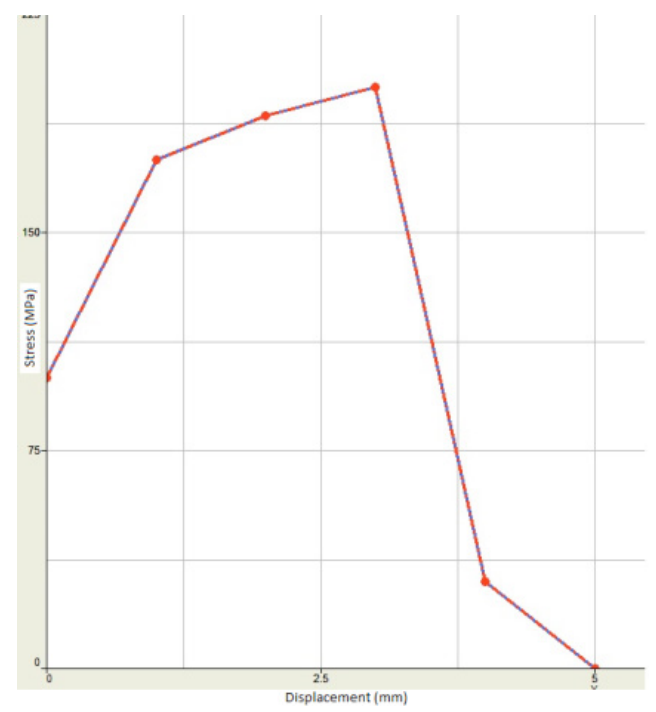

Figure 2. Example of stress displacement function.

The displacement failure criterion used in the type 2 interface, which measures the relative displacement for each connected node, is independent of the problem of small varying gaps. Each relative displacement is compared to the defined failure limit displacement. Element failure is dictated by the failure displacement criterion included in the interface definition. The above Figure 2 shows an example, stress/relative displacement function This function is used to calculate the transmitted loads between the slave and master segment. If the transmitted stress is smaller than the defined maximum stress the transmitted load is left unchanged. If the calculated maximum stress is larger than the defined maximum stress, the transmitted loads are reduced by the adjustment factor as mentioned below.

$\operatorname{Fac}_{N}=\min \left\{\sqrt{\frac{\sigma_{N_{\max }{ }^{2}}}{\max \left[\left(\sigma_{N}(t)\right) \cdot{ }^{2}, 10^{-20}\right]}}, 1\right\}$ 


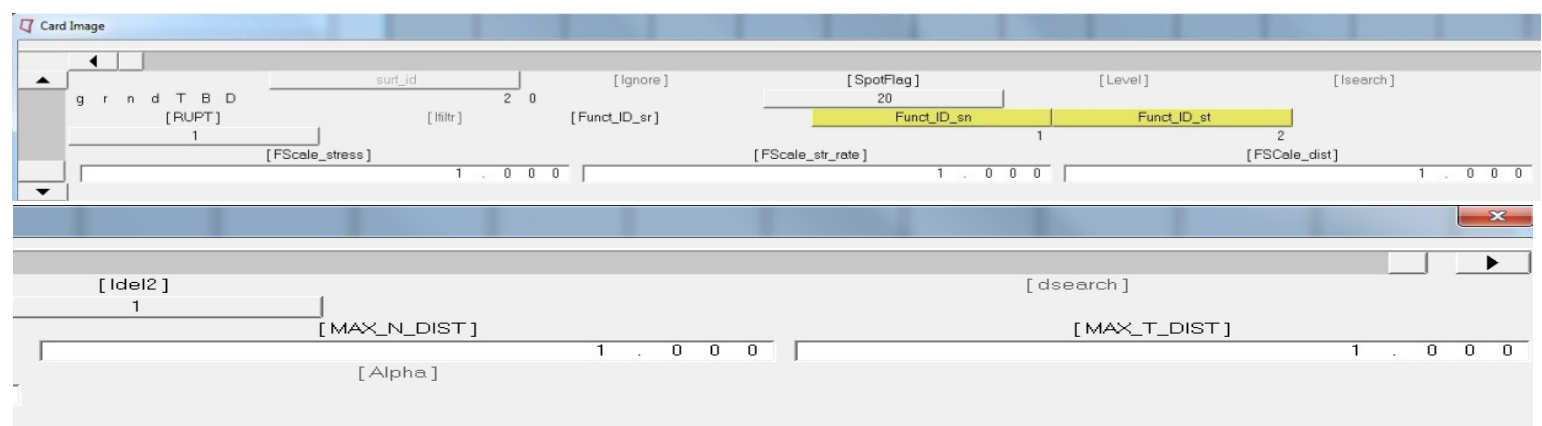

Figure 3. Example of RADIOSS rupture interface card definition.

Table 1. Johnson Cook plasticity parameters for plate and projectile

\begin{tabular}{|c|c|c|c|c|c|c|c|c|}
\hline Material & \multicolumn{2}{|c|}{ Density $\left(\frac{\mathbf{k g}}{\mathbf{m}^{3}}\right)$} & \multicolumn{2}{|c|}{ Poisson ratio } & \multicolumn{4}{|c|}{ Young's modulus (GPa) } \\
\hline Tungsten carbide & \multicolumn{2}{|c|}{14770} & \multicolumn{2}{|r|}{0.25} & \multicolumn{4}{|c|}{620} \\
\hline Steel 4030 & \multicolumn{2}{|c|}{7830} & \multicolumn{2}{|r|}{0.3} & \multicolumn{4}{|c|}{210} \\
\hline Aluminum & \multicolumn{2}{|c|}{2703} & \multicolumn{2}{|r|}{0.33} & \multicolumn{4}{|c|}{690} \\
\hline Material & A (Mpa) & B (Mpa) & $\mathrm{n}$ & $\mathrm{T}_{\text {mel }}(\mathrm{K})$ & $\mathrm{T}_{\text {ref }}(\mathrm{K})$ & $\mathrm{m}$ & c & $\mathrm{a}_{0}$ \\
\hline Tungsten carbide & 4000 & 93000 & 0.65 & 1768 & 293 & 1 & - & - \\
\hline Steel 4340 & 792 & 510 & 0.26 & 1793 & 293 & 1.03 & 0.014 & 1 \\
\hline Aluminum & 262 & 162.1 & 0.278 & 925 & 293 & 1.34 & - & - \\
\hline
\end{tabular}

$F a c_{T}=\min \left\{\sqrt{\frac{\sigma_{T_{\max }{ }^{2}}}{\max \left[\left(\sigma_{T}(t)\right) \cdot{ }^{2}, 10^{-20}\right]}}, 1\right\}$

The adjustment factor is applied to the transmitted forces in the next step to flatten the peaks resulting from the infinite stiffness of the interface. The element yields based on the rupture criteria corresponding to the interface. The following Figure 3, show a typical input card for the TYPE 2 interface.

\section{Finite Element Modeling of Projectile and Composite Armour Plate}

The $7.62 \mathrm{~mm}$ tungsten projectile is modeled using linear teta mesh as shown in the following Figure 4. A multilayer plate of size $50 \times 50 \mathrm{~mm}$ is modeled using linear hex mesh. This consists of two layers (each of $9 \mathrm{~mm}$ thick), namely, top layer made of steel and the bottom layer made of Aluminum. Two layers are held together forming multi layered composite structure. The present model is analyzed with different interface formulation using RADIOSS. The material model for plasticity and failure considered for the present analysis are as shown in the following Table 1 and Table 2.

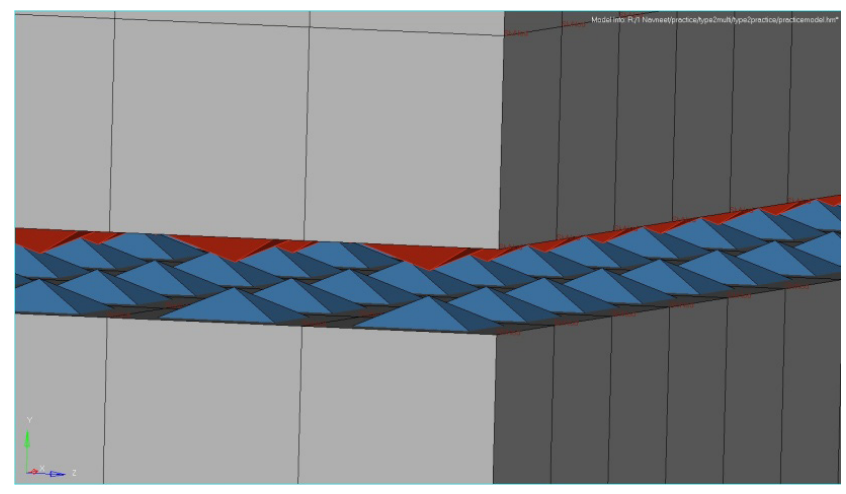

Figure 4. Finite element model with TYPE 2 interface surface.

Table 2. Johnson Cook failure parameters for plate and projectile

\begin{tabular}{lccccc}
\hline Material & $d_{1}$ & $d_{2}$ & $d_{3}$ & $d_{4}$ & $d_{5}$ \\
\hline Tungsten carbide & 0.0 & 0.0093 & -1.95 & 0.0 & 0 \\
Steel 4340 & 0.05 & 3.44 & -2.12 & 0.002 & 0.61 \\
Aluminum & -0.77 & 1.45 & -0.47 & 0.0 & 1.6 \\
\hline
\end{tabular}


Material data from $[6,17$ is considered in the present analysis. In all these simulations, it should be noted that the erosion criteria is kept on, which means the failed elements are deleted from the simulation. This is due to the fact that excessively distorted elements destabilize the time step. Also, it is to be noted that in physical firing, the eroded elements from the plate or projectile are basically burnt into flares (due to very high friction area) or disintegrate from the respective body. In either case they do not contribute to the energy of the system. Hence, deletion from the simulation will not violate the physical phenomenon.

\section{Results and Discussions}

In the first case, bonding is modeled using the TYPE 7 interface. In the second analysis, the TYPE 2 interface is considered. From the simulation, it is found that TYPE 2 interface alone is not sufficient to define the interaction between the multiple layers of the composite plate as it does not account for the contact beyond the failure of bonding. Here, it is to be noted that the TYPE 2 interface takes care of the bonding between the layers which on failure is broken. It does not consider the subsequent interactions between the layers after the failure of bonding. Hence a combination of TYPE 2 and TYPE 7 interface between the layers of the plate is considered for analysis. Further, it is to be noted that TYPE 7 interface is defined between the plate and projectile.

\subsection{TYPE 7 Interfaces between Two Surfaces}

In this case, two general purpose TYPE 7 interface is used, viz., between projectile and plate and second, between two layers of the plate. Initially, Aluminum layer is considered as master set and steel as slave nodes and vice versa is considered.

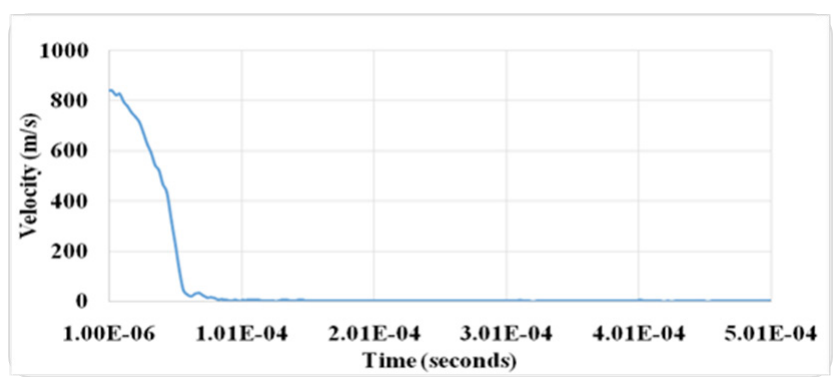

Figure 4. Velocity vs. Time plot for TYPE 7 interface between plates and modified tungsten carbide projectile.

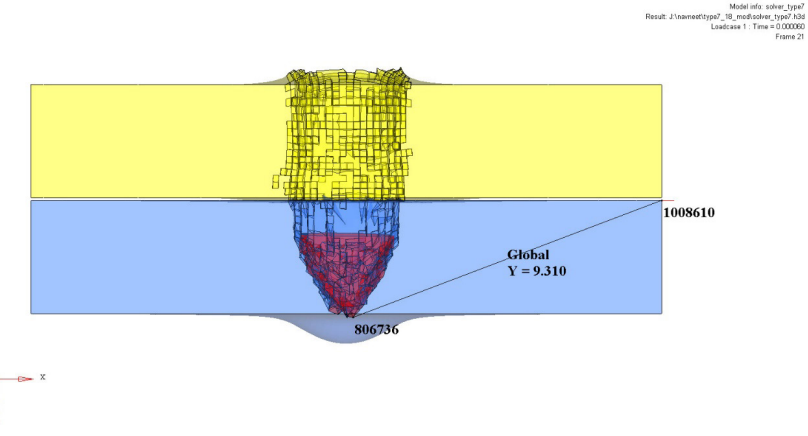

Figure 6. DOP at $60 \mu$ s for TYPE 7 interface between plates and modified tungsten carbide projectile.

Reduction in velocity with respect to time is shown in Figure 5. The above Figure 6 shows the position of projectile in $60 \mu \mathrm{s}$, when the velocity has reached $0 \mathrm{~m} / \mathrm{s}$. It is seen that the Depth Of Penetration (DOP) is $9.3 \mathrm{~mm}$, measured from the interface region between two metal layers.

\subsection{Combination of TYPE 7 and TYPE 2 Interface between Two Plates}

As discussed above, the mere TYPE 2 interface is insufficient to define the interface between the layers. Hence, a combination of TYPE 7 and TYPE 2 interface formulation is applied in the present analysis. A detailed study was made on the TYPE 2 interface wherein the effect of the stress, displacement curve was analyzed in detail. The magnitude of the curve was increased by several orders. From the analysis, it is found that, beyond a specific set of values, an increase in the order of the stressdisplacement curve does not influence the penetration results. In on the other hand states that the effect of resin on the depth of penetration is limited, beyond which no significant improvement in the penetration behavior is expected. With the optimal value, the analysis is done and the following results are obtained. From Figure 7 and 8 , it is seen that the velocity of projectile tends to zero in 57 microseconds. Further, $8.6 \mathrm{~mm}$ depth of penetration is measured from the plane of interface between two metal layers. 


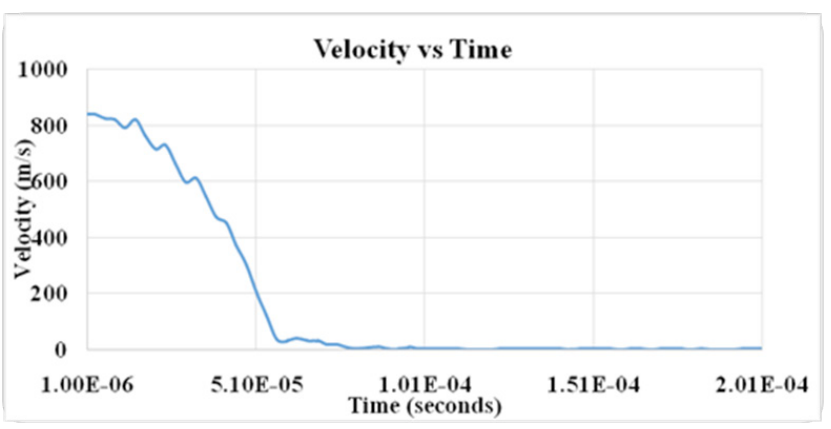

Figure 7. Plot for combination of TYPE 7 and TYPE 2 interface between plates and modified tungsten carbide.

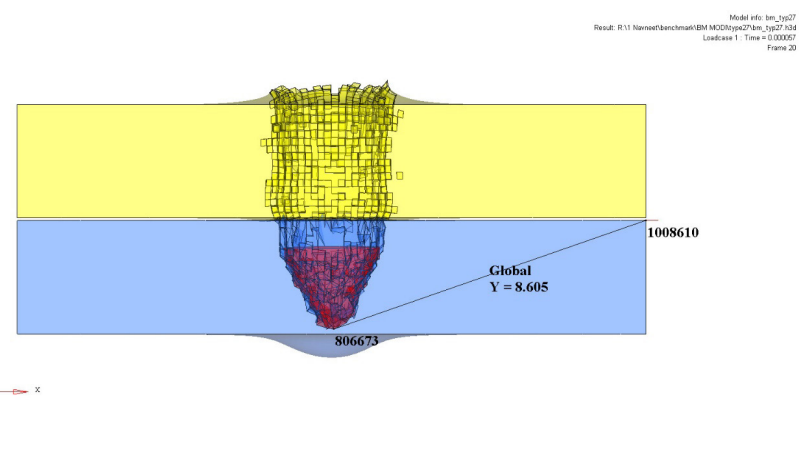

Figure 8. DOP at $57 \mu$ s for combination of TYPE 7 and TYPE 2 interface between plates and modified tungsten carbide projectile.

From the two analyses, it is observed that the event time is marginally reduced by $3 \mu$ s. Further, it is to be noted that the projectile has travelled $0.6 \mathrm{~mm}$ more when using TYPE 7 interface than the second case with a combination of TYPE 2 and TYPE 7 interface which is not significant in this study. But in case of the second analysis, the delamination is considerably lesser when compared to the first analysis with only TYPE 7 contact. Hence, from this analysis, it is seen that TYPE 2 interface can be applied as a replacement for complete modeling of resin bonding. Further, it helps in capturing the delamination that happens during penetration.

\section{Conclusion}

The present study has been carried out for small arm projectile penetrating the armor. The analysis focused on the impact of $7.62 \mathrm{~mm}$ diameter Tungsten Carbide projectile on a multilayer composite plate made of Aluminium and Steel. Hyper Works software tool is used to simulate the phenomenon wherein Hyper Mesh used for preprocessing and RADIOSS as a solver. It is to be noted that the elements are deleted as it gets disintegrated from the main projectile. This is adequate, as the disintegrated elements do not contribute to the energy of the projectile or deformation in plates and hence cannot affect the results.

Further, the present work focused on the study of the different interface formulation for multi material layer plate. Interface formulation is attempted with TYPE 7 and TYPE 2 interfaces available in RADIOSS solver. The effect of adhesives is specifically simulated by TYPE 2 interface. It is observed that the depth of penetration is not significantly affected due to the TYPE 2 interface. However, the delamination behavior of the layers is captured in the TYPE 2 interface when compared to TYPE7.

Even though, the modified interface with combination of TYPE 2 and TYPE 7 does not influence the depth of penetration when compared to the TYPE 7 interface, more studies needs to be done with respect to delamination. This, in particular, influences in the study of multiple hit of projectiles. Hence, it is concluded that TYPE 7 formulation gives considerably acceptable results for capturing the depth of penetration in case of multilayer composite structures. TYPE 2 interface has to be studied in cases where delamination has influence, for example in multiple hits of projectiles etc.

\section{References}

1. Piekutowski AJ, Forrestal MJ, Pooron KL, Warren TL. Perforation of aluminum plates with Ogive-nose steel rods at normal and oblique impacts. International Journal of Impact Engineering. 1996; 18(1):7-8.

2. Dokainish DK, Subbaraj K. A survey of direct time-integration methods in computational structural dynamics-II. Explicit Methods, Computers and Structures. 1989; 32(6):1371-86.

3. Subbaraj K, Dokainish MA. A survey of direct time-integration methods in computational structural dynamics-II. Implicit Methods, Computers and Structures. 1989; 32(6):1387-401.

4. Gordon R, Johnson J, Cook WH. A constitutive model and data for metals subjected to large strains, high strain rates and high temperature. Internal Report. 1997. p. 1-17.

5. Lindgren L, Wedberg D. Material modeling and physical based models with particular emphasis on high strain rates. Internal Report. 2006. p. 1-15.

6. William F, Hosford H. Mechanical Behavior of Materials. UK: Cambridge University Press; 2005. 
7. Gooch WA. An overview of ceramic armour applications. Ceramic Transections. 2002; 134(2):3-21.

8. Schwer L. Optional strain-rate forms for the Johnson Cook Constitutive Model and the role of the parameter Epsilon_01. 6th European LS-DYNA Users' Conference; USA. 2016. p. 1-17.

9. Hubert W, Meyer M, David S, Kleponis K. An analysis of parameters for the Johnson-Cook strength model for 2-inthick rolled homogeneous armor, PN. 2001.

10. Dorogoya A, Rittel D. Determination of the Johnson-Cook material parameters using the SCS specimen. Internal Report. 2009; 49(1):881-5.

11. Help Document, Altair Engineering, Inc. 2015. p. 1-7.

12. Arad M, Touati D, Latovitz I, The relation between initial yaw and long rod projectile shape after penetrating an oblique thin plate. 4th LSDYNA European user Conference; 2013. p. 1-12.
13. Lanzerath $\mathrm{H}$, Nowack N, Mestres E. Simulation tool including failure for structural adhesives in full-car crash models. EHTC Conference; 2008. p. 1-7.

14. Simulation of the ballistic perforation of aluminum plates with Abaqus/Explicit. Abaqus Technology. 2012 Jul:1-6.

15. Narayanamurthy V, Rao CL, Rao BN. Numerical simulation of ballistic impact on armour plate with a simple plasticity model. Defence Science Journal. 2014; 64(1):1-324.

16. Borvik T, Dey S, Clausen AH. Perforation resistance of five different high strength steel plates subjected to small-arms projectiles. International Journal of Impact Engineering. 2009; 36(5):948-64.

17. Deniz T. Ballistic penetration of hardened steel plates [Thesis]. The Graduate School of Natural and Applied Sciences. Middle East Technical University; 2010. p. 1-133. 